\title{
Effect of Pupil Size on Wavefront Refraction during Orthokeratology
}

\author{
Miguel Faria-Ribeiro*, Rafael Navarro*, and José Manuel González-Méijome*
}

\begin{abstract}
Purpose. It has been hypothesized that central and peripheral refraction, in eyes treated with myopic overnight orthokeratology, might vary with changes in pupil diameter. The aim of this work was to evaluate the axial and peripheral refraction and optical quality after orthokeratology, using ray tracing software for different pupil sizes.

Methods. Zemax-EE was used to generate a series of 29 semi-customized model eyes based on the corneal topography changes from 29 patients who had undergone myopic orthokeratology. Wavefront refraction in the central 80 degrees of the visual field was calculated using three different quality metrics criteria: Paraxial curvature matching, minimum root mean square error (minRMS), and the Through Focus Visual Strehl of the Modulation Transfer Function (VSMTF), for 3- and 6-mm pupil diameters.

Results. The three metrics predicted significantly different values for foveal and peripheral refractions. Compared with the Paraxial criteria, the other two metrics predicted more myopic refractions on- and off-axis. Interestingly, the VSMTF predicts only a marginal myopic shift in the axial refraction as the pupil changes from 3 to $6 \mathrm{~mm}$. For peripheral refraction, minRMS and VSMTF metric criteria predicted a higher exposure to peripheral defocus as the pupil increases from 3 to $6 \mathrm{~mm}$.

Conclusions. The results suggest that the supposed effect of myopic control produced by ortho-k treatments might be dependent on pupil size. Although the foveal refractive error does not seem to change appreciably with the increase in pupil diameter (VSMTF criteria), the high levels of positive spherical aberration will lead to a degradation of lower spatial frequencies, that is more significant under low illumination levels.
\end{abstract}

(Optom Vis Sci 2016;93:00-00)

Key Words: pupil size, peripheral refraction, wavefront refraction, orthokeratology, visual metrics

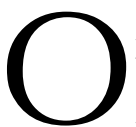
rthokeratology (ortho-k) changes the ocular refraction by the programmed application of reverse geometry rigid gas-permeable contact lenses. To correct myopia, the central cornea is flattened to induce a reversible change on the epithelial thickness profile. The central epithelial layer thins and the front surface corneal power decreases over the central 4 to $5 \mathrm{~mm}$ central zone. The paracentral zone (transition zone) of 1.5 to $2.0 \mathrm{~mm}$ surrounding the central zone increases in curvature, in a direct relationship with the amount of central flattening needed to correct the myopic refractive error. ${ }^{1}$

With the advent of highly permeable materials, overnight ortho-k has become an effective and safe mode of vision correction for moderate and low myopia and was approved by the United Sates

\section{${ }^{*} \mathrm{PhD}$}

Clinical \& Experimental Optometry Research Lab (CEORLab), Centre of Physics, School of Sciences (Optometry), University of Minho, Braga, Portugal (MF-R, JMG-M); and Instituto de Ciencia de Materiales de Aragón, Consejo Superior de Investigaciones Científicas-Universidad de Zaragoza, Zaragoza, Spain (RN).
Food and Drug Administration in 2002. ${ }^{2}$ Over the last decade, systematic research reports, including randomized and controlled clinical trials, confirmed that ortho-k reduces the rate of axial length increase by 40 to $60 \%$ in children when compared with single vision spectacles or contact lenses. ${ }^{3}$ Ortho-k is currently one of the most effective optical strategies of myopia control and is at present the modality with the largest volume of accumulated evidence relating to the efficacy to regulate myopia progression in children. ${ }^{3,4}$

Previous research has explored potential predictors of the myopia regulation effect with ortho-k. Cho et al. ${ }^{5}$ found a moderate correlation between the treatment target and the regulation effect. In their cohort of ortho-k lens wearers aged from 6 to 10 years, higher myopes had a lower axial elongation over a 2-year period, whereas the opposite was found in a spectacle control group. This result raises the hypothesis that the greater the corneal reshaping effect, the higher the regulation efficacy, probably as a result of greater peripheral myopic defocus. ${ }^{6,7}$ However, these trends have not been confirmed in other similar studies after 2 and 5 years, ${ }^{8,9}$ including a controlled and randomized study. ${ }^{10}$ Despite the correlation between treated myopia 
and peripheral myopic shift of about $1: 1$ seen in ortho-k eyes, ${ }^{11}$ this relationship gets complicated by the significantly different eye shapes and meridional asymmetries seen in myopic eyes. ${ }^{12,13}$

Recently, an association was found ${ }^{14}$ between pupil size and myopia control effect in eyes treated with orthokeratology. Larger pupil diameters were associated with higher control effect, hypothetically as a result of a larger retinal area being exposed to the peripheral myopic defocus. This effect might come as a consequence of the peripheral increase in corneal curvature induced by these treatments, and it is expected to vary depending on the area of the cornea flattened by the lens treatment zone. However, larger pupil size might also change the pattern of relative peripheral refraction, either sphere or cylinder, and contribute to the difference in regulation effects found. Thus, the theoretical evaluation of the effect of the pupil size on the effective optical focusing properties of the eye seems to be relevant to improve our understanding of the working principles and efficacy of such treatments.

Most autorefractors used to assess the effect of ortho-k treatment are limited to a measured annular zone of approximately 2.0 to $3.0 \mathrm{~mm}^{15-17}$ irrespective of the actual pupil size of the patient. Modern aberrometers normally use two different criteria to estimate refraction from wavefront data. One approach, called Zernike refraction, specifies the vergence of a point source that focuses a "disk of least confusion" into the image plane, defined by the retinal layer where the aberrometer's probe beam reflects. The second approach, Paraxial refraction, specifies the vergence of a point source that focuses paraxial rays into the plane of reflection of the aberrometer's probe beam. ${ }^{18}$ In the absence of higher-order aberrations, Zernike and Paraxial refractions are identical, but in eyes such as the ones treated with ortho-k, the high levels of positive fourth-order spherical aberration, and other higher orders, ${ }^{19,20}$ may bias these metrics in different ways. ${ }^{21}$ Zernike defocus will tend to yield more myopic refractions as the pupil becomes larger, due to increased contribution of positive spherical aberration. Paraxial refraction, by definition, will not change with pupil diameter if sufficient higher-order terms are used in the calculation. Thus, none of these methods may be robust enough to obtain an unbiased estimation of refraction, ${ }^{22}$ especially for large field angles. Considering that all the information regarding refraction and quality of vision in the periphery is derived from instruments optimized to measure axial refraction, we hypothesize that ray-tracing could be used to isolate the contribution of the different optical elements of the eye and bypass some of the encountered limitations in peripheral aberrometry. ${ }^{23}$

The present study aims to test the hypothesis that changes in pupil size induce changes in the pattern of axial and peripheral refraction and peripheral optical quality using ray tracing software. To this end, a fundamental aspect is to find the most appropriate metric for estimating the refractive errors from the wavefronts computed in our partially customized eye models.

\section{MATERIALS AND METHODS}

\section{Ortho-k Patients and Measurements}

Twenty-nine patients (age $24 \pm 5$ years) were fitted with Corneal Refractive Therapy (Paflucon D, Paragon CRT) contact lenses for 9 to 12 months to correct myopia between -1.00 and
$-5.75 \mathrm{D}$ (mean $\pm \mathrm{SD}=-3.62 \pm 1.11 \mathrm{D})$ with refractive astigmatism below 1.50D. Paragon CRT Dual Axis was used in subjects with limbus-to-limbus corneal astigmatism. The initial contact lenses were fitted following the nomograms of adaptation of the corneal refractive therapy manufacturer. If needed, some contact lens parameters were changed to obtain a full correction of the myopic refraction and, at the same time, a well-centered treatment. Trial lenses were derived from sliding table nomograms provided by the manufacturer, which have shown high levels of predictability in terms of first trial success. ${ }^{24}$ Fitting was evaluated according to the recommendations of the manufacturer regarding fluorescein pattern, topographical evaluation, and refractive and visual outcomes. Parameters of the corneal refractive therapy lenses were as follows: base curve radius (mean $\pm \mathrm{SD}$ [minimum, maximum] $)=8.22 \pm 0.49 \mathrm{~mm}[7.80,8.80 \mathrm{~mm}]$, return zone depth $=530.80 \pm 19.32 \mu \mathrm{m}[500,575 \mu \mathrm{m}]$, and landing zone angle $=31.45 \pm 0.88^{\circ}\left[31.00^{\circ}, 34.00^{\circ}\right]$. These refer to the final parameters of lenses worn by patients, not necessarily the first trial lenses.

All the enrolled subjects were able to achieve logMAR 0.0 visual acuity without any further compensation. Individual data from anterior elevation topography of each patient's left eye was obtained using Medmont E300 corneal topographer (Medmont, Victoria, Australia), with pupil center determined by the topographer as reference. All patients attending the measurement visit were wearing the lenses overnight for at least three consecutive days. Changes in morphology, topography, and optics after ortho$\mathrm{k}$ can be found in another paper. ${ }^{25}$ All procedures were performed according to the Declaration of Helsinki. Approval for the study was obtained from the ethics committee of University of Minho School of Sciences.

\section{Semi-Customized Eye Models}

Zemax-EE numerical ray tracing software was used to create a series of 29 semi-customized eye models based on the Navarro eye model. ${ }^{26}$ The front surface of the cornea of that initial generic eye model was replaced by the Zernike Standard Sag surface ${ }^{27}$ computed from the elevation data of each patient. This surface includes a regular revolution conic surface plus a Zernike polynomial expansion, which accounts for departures of the real surface from the regular basis. ${ }^{28}$ The individual data were fitted to a Zernike Standard Surface equation by a least-squares method implemented in Matlab (The MathWorks, Natick, MA). The same internal optics were used in all the semi-customized eye models to isolate the contribution of the ortho-k treatments. The vitreous length of each model eye was optimized for central vision according to the Paraxial focus metric criteria. This was accomplished by minimizing the root mean square (RMS) wavefront error with respect to the centroid, at a visual field of 0 degrees, of Zemax's default merit function, using a small entrance pupil diameter of $0.1 \mathrm{~mm}$.

\section{Wavefront Error}

Into-the-eye ray trace was performed along the central 80 degrees of the horizontal field, sampled in 10-degree steps, at a reference wavelength of $555 \mathrm{~nm}$ with entrance pupil diameters of 3 and $6 \mathrm{~mm}$. Zemax software can provide wavefront $W(x, y)$ from 
the optical path differences. Optical path difference is calculated by tracing a bundle of rays passing through a grid of points $(x, y)$ on the exit pupil plane. The effective pupil is a circle on-axis and approximately elliptical off-axis. Zemax also provides a modal representation of the wavefront expressed in terms of standard Zernike polynomials. ${ }^{27}$ However, because Zernike aberrations can be derived only for circular pupils, the software stretches the offaxis elliptical pupil along its minor axis into a circular form, by a factor equal to its aspect ratio (minor diameter/major diameter). Using this method, Zernike coefficients were calculated up to sixth order and reported using the Optical Society of America standard. ${ }^{29}$ Both representations of the wavefront, raw data (values on a $512 \times 512$ samples grid) and modal (Zernike coefficients), were exported to Matlab for further processing.

\section{On- and Off-Axis Refraction from Wavefront Data}

The refractive state of the eye can be measured by subjective or objective methods. Although it is unknown which criteria the human eye actually uses for focusing, and as such the ideal optimization method is yet to be determined, several metrics have been used to estimate refraction from wavefront data. ${ }^{18,30,31}$ When higher-order aberrations are significant, image plane quality metrics such as the Visual Strehl ratio computed in frequency domain (MTF method) (VSMTF) seem to be less biased by the high levels of spherical aberration. ${ }^{31,32}$ This metric takes into account that different frequencies respond differently to defocus and neural sensitivity varies with frequency ${ }^{33}$ in accordance to visual channel theory, which establishes that the visual pathway decomposes light in frequency bands. ${ }^{34}$

In this work, we calculated foveal and peripheral refraction from wavefront data, obtained by ray tracing, using the following metrics:

\section{Zernike and Paraxial Refraction}

For eccentric fields, i.e. elliptical pupils, Zemax stretches the wavefront along the minor axis into a circular pupil to fit the wavefront optical path differences with circular Zernike polynomial. Such stretching affects all the Zernike coefficients. ${ }^{35}$ Recently, Zernike-like orthogonal polynomials were proposed for elliptical pupils. ${ }^{36,37}$ Nevertheless, here we were mainly interested in computing the refractive error, so we used the equations provided by Atchison et al. ${ }^{38}$ truncated at second order for Zernike refraction and up to sixth order for Paraxial refraction. Because Zemax calculates the approximate shape of the wavefront at the exit pupil as seen from the on-axis chief ray image point, ${ }^{27}$ we implemented an improvement that may be important for large field angles due to pupil aberrations: Instead of assuming that the minor axis of the off-axis pupil shortens by a factor equal to the cosine of the field angle $(\Theta)$, we calculated the actual aspect ratio of the wavefront at the exit pupil. For the sake of simplicity, there is no need to alter the original equations. The proper correction can be accomplished by substituting $\Theta$ in the original equations by the inverse cosine of the aspect ratio of the exit pupil. Validation of this approach was performed with a Matlab script written to stretch the wavefronts imported from Zemax along their minor axis in to a circular form, fit the optical path differences with Zernike circular polynomials, and compare the obtained coefficients with the ones computed in Zemax. All differences between coefficients were below $0.005 \mu \mathrm{m}$, justifying the validity of the approach.

\section{VSMTF Trough Focus Refraction}

The other approach used to calculate wavefront refraction was similar to the one previously described by Guirao and Williams. ${ }^{39}$ Detailed methodology can be found in the cited paper. The procedure executes a search in a three-dimensional space, finding the values of sphere, cylinder, and axis of the correcting lens that yields the maximum value of a visual quality metric. This was achieved computationally by adding to the computed wavefronts a series of defocused spherical and cylindrical wavefronts that simulate the trial lenses employed during a subjective refraction examination. In summary, this procedure finds the sphericalcylindrical wavefront which, when added to the ocular wavefront obtained by ray tracing, optimizes the eye model retinal image quality accordingly to the VSMTF objective metric criteria:

$$
V S M T F=\frac{{ }_{-\infty}^{\infty} \int_{-\infty}^{\infty} \int \operatorname{CSF}_{\mathrm{N}}(\mathrm{fx}, \mathrm{fy}) \cdot \operatorname{MTF}(\mathrm{fx}, \mathrm{fy}) \mathrm{dfxdfy}}{{ }_{-\infty}^{\infty} \int_{-\infty}^{\infty} \operatorname{CSSF}_{\mathrm{N}}(\mathrm{fx}, \mathrm{fy}) \cdot \operatorname{MTFDL}(\mathrm{fx}, \mathrm{fy}) \mathrm{dfxdfy}}
$$

The quick contrast sensitivity functions $(q C S F)$ curves measured by Rosén et al..$^{40}$ at 20 degrees of the nasal and temporal visual fields were used (courtesy of Linda Lundström) to derive the Neural Contrast Sensitivity Functions $\left(C S F_{N}\right)$ for each peripheral location. The qCSFs for 20 degrees nasal and temporal visual fields were $\mathrm{M}$-scaled for the other peripheral locations according to the cortical magnification factor $(M)$ equations provided by Rovamo and Virsu, ${ }^{41}$ and applied in Eq. 1 as general population models of the peripheral $C S F_{N}$.

\section{Astigmatic Off-Axis Refraction}

Sphero-cylindrical refractions were converted to spherical equivalent $(M)$, with or against-the-rule astigmatism $\left(J_{0}\right)$ and oblique astigmatism $\left(J_{45}\right),{ }^{42}$ and applied in the following equations to calculate the off-axis tangential $\left(F_{T}\right)$ and sagittal $\left(F_{S}\right)$ power errors, considering clinical refractive notation with negative cylinder:

$$
\begin{gathered}
F_{T}=M+J_{0} \\
F_{S}=M-J_{0}
\end{gathered}
$$

$F_{S}$ and $F_{T}$ components represent, in this case, the dioptric vergence required to correct the power error in the radial and sagittal meridians along of the horizontal visual field $\left(J_{45}=0\right)$. It is worth mentioning that all subjects were treated as stigmatic by removing the on-axis $J_{0}$ and $J_{45}$ values for all field positions. This way, we isolated the effect of off-axis oblique astigmatism from foveal astigmatism.

All the procedures were repeated for entrance pupils' diameters of 3 and $6 \mathrm{~mm}$. The entrance pupil position and center was interactively calculated by Zemax's robust ray-aiming algorithm. 
Matlab scripts and a Zemax macro were written to automatize all the procedures and export the values into data sheets.

\section{Best Metric Criteria}

To establish which metric predicts the best foveal refraction, we computed the image quality for each eye model with 3-and 6-mm pupils. The wavefront error maps of each patient, at nine different visual field angles and two pupil diameters, were exported from Zemax to Matlab as $512 \times 512$ matrices. Each wavefront matrix was used to compute the point-spread function, and the optical transfer function, using standard Fourier optics methods. The point-spread functions for 0 degrees of visual field were convolved with an eye chart template to simulate the retinal image.

Two random subsets of 29 images, correspondent to these simulated images optimized according to each of the three quality metrics described above, for 3- and 6-mm pupil diameters, were subjectively evaluated by three well-corrected, experienced observers. The observers were blinded to the metric criteria used and asked to grade with a score between 5 and 0 with terms for general guidance (Excellent, Good, Fair, Poor, Bad) each of the images, presented in a 13-inch computer screen at a $50 \mathrm{~cm}$ distance under normal office illumination conditions. Each of the computed images was presented together with a second image of a perfect non-aberrated model eye for reference. A similar procedure has been used recently to grade the image quality generated by multifocal lenses by Rio and Legras. ${ }^{43}$

\section{Statistical Analysis}

Statistical analysis was conducted using SPSS v21.0 program (IBM Inc., Chicago, IL). All data are reported as mean and standard deviation unless otherwise stated. Paired t-tests were used to compare the differences between the three metrics $\left(F_{S}, F_{T}\right.$, and $M$ refraction components, and the aberration Zernike coefficients for primary horizontal coma and spherical aberration), for 3- and 6 -mm pupils, at all field angles. A p value $<0.05$ was considered statistically significant.

\section{RESULTS}

Fig. 1 shows the refraction profiles across 80 degrees of visual field along the horizontal meridian for $3-\mathrm{mm}$ and $6-\mathrm{mm}$ pupil diameters, calculated using three different metric criteria. The vitreous length of each eye model was adjusted for emmetropia using the Paraxial metric criteria. As a consequence, the eye models present an ametropic condition for the other two metric criteria, with more myopic axial refractions $(-0.47 \pm 0.28 \mathrm{D}$ and $-0.69 \pm 0.42 \mathrm{D}$ for the VSMTF and minRMS, respectively, for a $3-\mathrm{mm}$ pupil). As the pupil dilates from 3 to $6 \mathrm{~mm}$, Paraxial metric criteria predicts a hyperopic shift in axial refraction (difference $=+0.25 \pm 0.17 \mathrm{D} ; \mathrm{p}<0.01$ ). The other two metrics predicted myopic shifts in axial refraction as the pupil dilates from 3 to $6 \mathrm{~mm}$, but although the minRMS metric predicts a significant myopic shift in axial refraction (difference $=-2.66 \pm 0.68 \mathrm{D}$; $\mathrm{p}<0.001$ ), interestingly, the VSMTF predicts only a marginal myopic shift (difference $=-0.03 \mathrm{D} ; \mathrm{p}=0.043$ ), which is consistent with experimental findings. ${ }^{44}$
As for peripheral refraction, the three metrics refractive components $F_{S}, F_{T}$, and $M$ present statistically significant differences between pupil sizes for almost all field angles except for the VSMTF metric $F_{T}$ component at -40 and 40 degrees $(p=0.18$ and $p=0.09$, respectively), for the Paraxial metric $F_{T}$ component at 20 degrees $(\mathrm{p}=0.41)$ and $M$ component at -20 degrees $(\mathrm{p}=0.08) . F_{S}, F_{T}$, and $M$ refractive components also show a strong significant correlation between 3-and 6-mm pupil diameters ( $\mathrm{r} \geq 0.7 ; \mathrm{p}<0.05$ except for the locations mentioned above), for all of the three metrics, with the 6-mm pupil refractive components always being more negative when calculated using the VSMTF and minRMS metric criteria. As expected, the correlation between the refractive components calculated for 3-and 6-mm pupil diameter were higher $(\mathrm{r} \geq 0.97 ; \mathrm{p}<0.01)$, except for $F_{S}$ at 10 degrees $(r=0.86 ; p<0.01)$, when the Paraxial criteria was used. In theory, Paraxial refraction should be independent of pupil size. This lack of a perfect correlation, along with a small but significant difference ( $\mathrm{p}<0.01$ for all angles except for $M$ at $-20^{\circ}$ and $F_{T}$ at $20^{\circ}$ ) in the peripheral refractive pattern between the two pupil sizes, may be due to the non-inclusion of higher (than sixth) order terms, such as eighth order spherical aberration, in the calculations.

The change in the peripheral refraction pattern, associated to the increase in pupil diameter, is most substantial when using the minRMS than with the VSMTF and Paraxial metrics. The peripheral refractive profile of the minRMS metric seems to reflect the refractive contribution of the more peripheral zones of the cornea as the visual angle increases. There is a clear myopic shift in the most central visual fields (more light is refracted by the more curved transition zone) that decreases for more peripheral angles as the contribution of the flatter peripheral zone of the cornea increases. Curiously, this is the only metric of the three that predicts a decrease in oblique (off-axis) astigmatism as the pupil increases (the shift in $F_{S}$ is greater than the shift in $F_{T}$, decreasing the interval of Sturm). The previous pattern is less evident when the $V S M T F$ metric is used. This is due to the nature of the peripheral $C S F$. As the visual field increases, the peripheral CSF gives more emphasis to the lower spatial frequencies of the $M T F$, which are optimized by a more negative lens. ${ }^{21}$

Peripheral refraction $M$ component presents a strong correlation with baseline axial refraction $\left(M_{\text {baseline }}\right)(0.79>\mathrm{r}>0.60 ; \mathrm{p}<0.001$ at $\pm 40^{\circ}$, for the three metric criteria and both pupil sizes). Despite these strong correlations, higher myopes will experience more peripheral defocus for both pupil sizes, thus the shift in peripheral refraction with the increase in pupil diameter will not be dependent of baseline axial refraction $\left(0.05>r>0.003 ; p>0.5\right.$ at $\pm 40^{\circ}$, for the three metric criteria and both pupil sizes).

Fig. 2 shows the pattern of primary horizontal coma and spherical aberration coefficients for both pupil sizes. The reverse in slope seen in the primary coma near $\pm 20^{\circ}$ seems to be due to the sudden decrease in power, corresponding to the transition from the more curved paracentral zone to the flatter peripheral zone. It can be seen from the curve that the treatments are slightly asymmetric and slightly decentered to the temporal side. Third-order horizontal coma showed significant differences between the 3 - and 6-mm pupil size with the exception of values around zero-crossings. Difference was maximum at -40 degrees of the nasal visual field $(1.24 \mu \mathrm{m} ; \mathrm{p}<0.001)$ and lower at the center $(0.034 \mu \mathrm{m}, \mathrm{p}>0.017)$.

Fourth-order spherical aberration presented statistically significant differences between 3- and 6-mm pupil size for all locations 

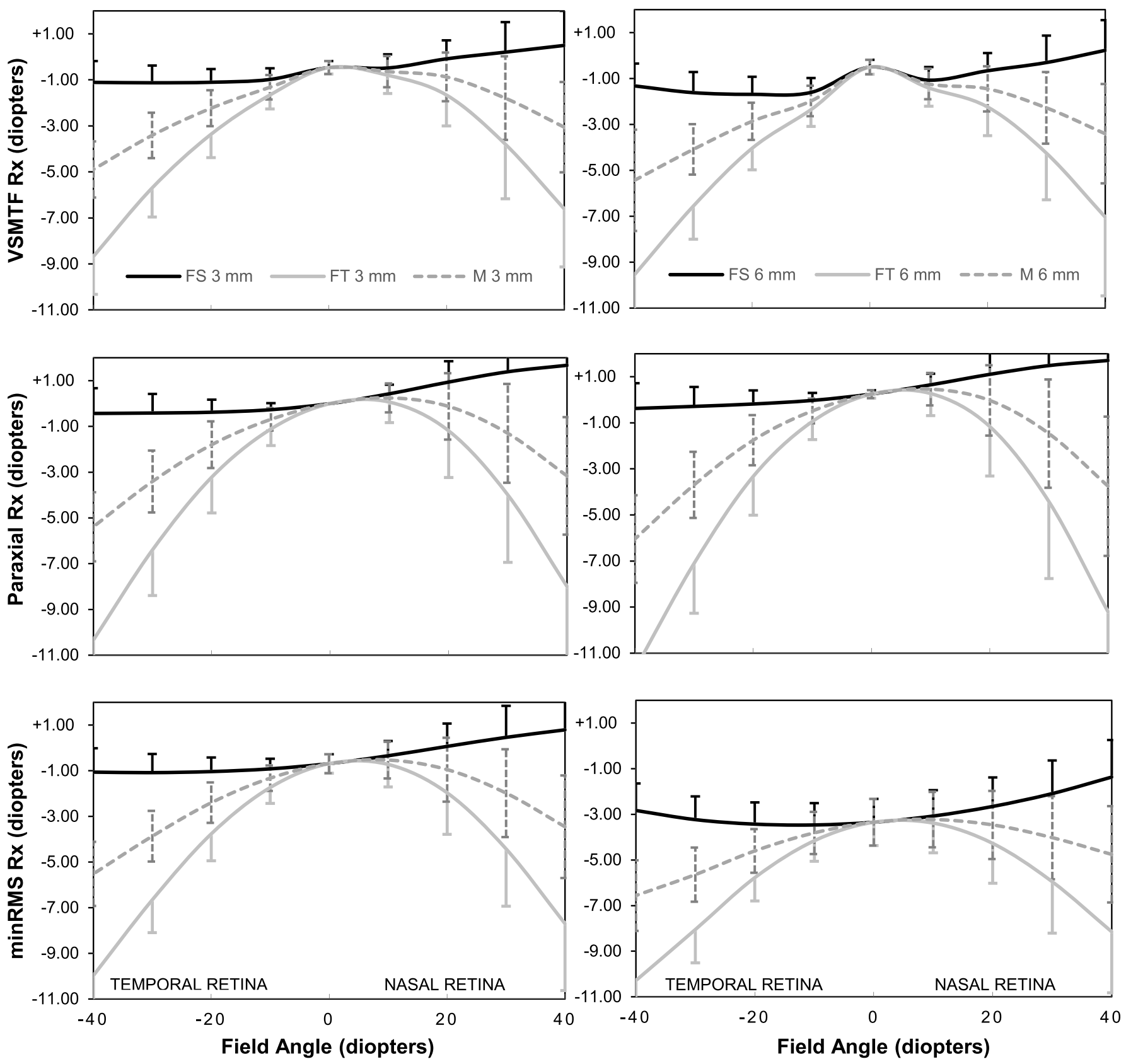

\section{FIGURE 1.}

Axial and peripheral refraction for 3-mm (left column) and 6-mm (right column) pupil diameters, across 80 degrees of visual field along the horizontal meridian calculated using the through focus visual Strehl of the modulation transfer function (VSMTF) (top), Paraxial (middle), and minimum root mean square error (minRMS) (bottom) metrics. Negative values of eccentricity represent the temporal retina (nasal visual field) and positive values represent the nasal retina (temporal visual field). Error bars represent one standard deviation. FS, sagittal power error; $F T$, tangential power error; $M$, spherical equivalent.

measured $(\mathrm{p}<0.001)$. Difference was maximum at 0 degrees $(0.95 \mu \mathrm{m} ; \mathrm{p}<0.001)$ and lower for the \pm 40 -degree locations $(0.29$ and $0.20 \mu \mathrm{m}$, respectively; $\mathrm{p}<0.001)$. Spherical aberration for a $6-\mathrm{mm}$ pupil diameter presents values almost four times higher than the ones encountered in untreated eyes.

Fig. 3 shows the average rating of the two sets of images graded by the observers to derive a quantitative (though subjective) information on which metric would perform better for foveal vision. According to our observers, there are only small differences in the perceived image quality for a 3-mm pupil, between the minRMS and the VSMTF. The Paraxial metric clearly shows the worst performance. As for a 6-mm pupil, the VSMTF is clearly superior to both Paraxial and $\min R M S$ quality metrics. For the larger pupil size, Paraxial metric was graded higher than the minRMS metric, as opposed to the result found with the smaller pupil.

The visual quality degradation (foveal vision) as a result of the increase in higher-order aberrations as a consequence of the increased pupil diameter can be seen in the convolved images of Fig. 4.

\section{DISCUSSION}

With this study, we aimed to verify the hypothesis that central and peripheral refraction, in eyes treated with myopic overnight orthokeratology, might suffer variations with changes in pupil 


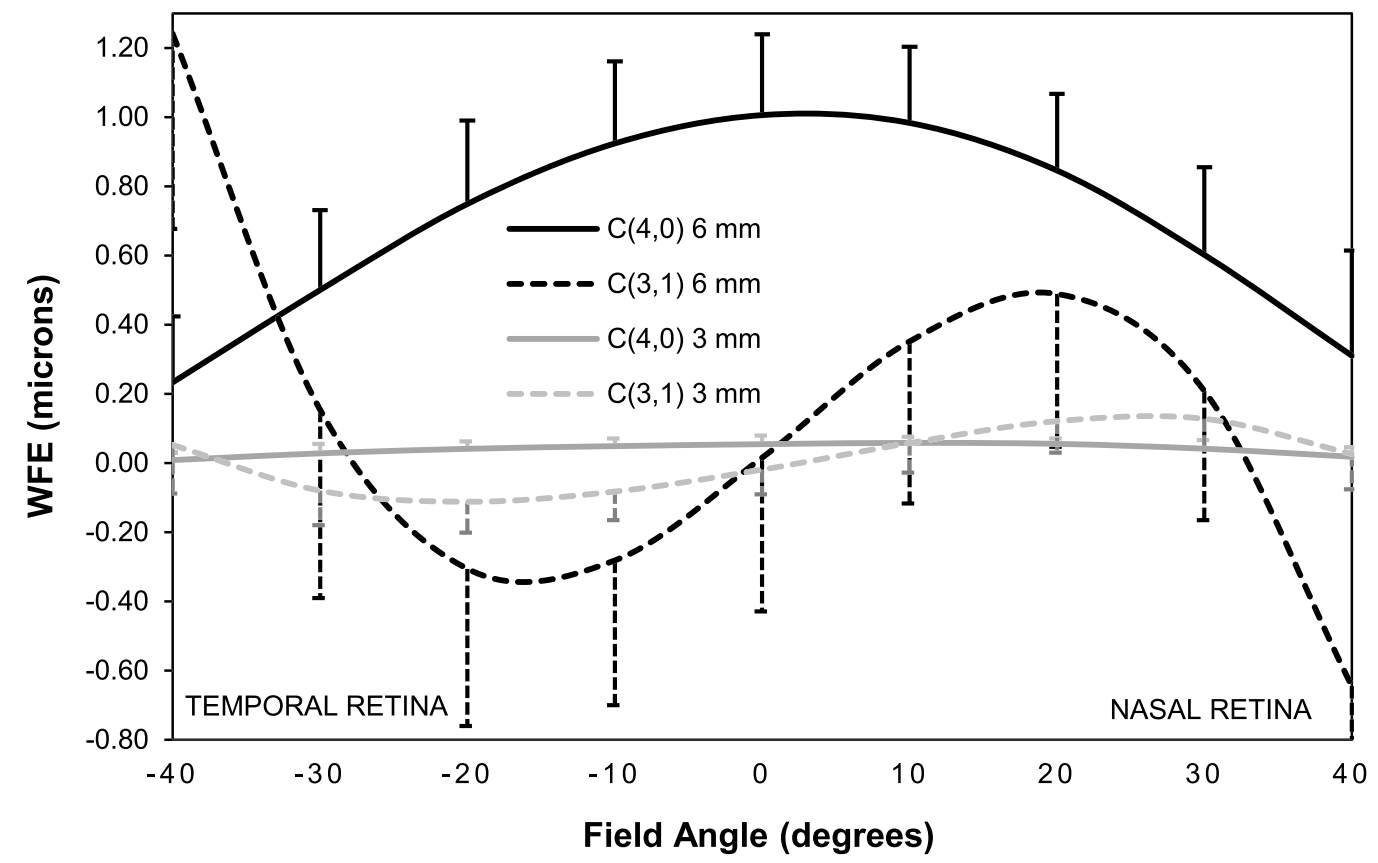

FIGURE 2.

Primary Zernike spherical aberration $\left(\mathrm{C}_{4}{ }^{0}\right)$ and horizontal coma $\left(\mathrm{C}_{3}{ }^{1}\right)$ for the 80 degrees of visual field along the horizontal meridian, for both pupil sizes. Negative values of eccentricity represent the temporal retina (nasal visual field) and positive values represent the nasal retina (temporal visual field). Error bars represent one standard deviation.

diameter. It has been suggested that, in the presence of primary spherical aberration, conventional measurements of subjective refraction closely match the ones predicted by the Paraxial refraction metric, largely because this is optimal for objects whose spatial frequency spectrum is dominated by high frequencies, such as small letters. ${ }^{44}$ Subjective grading of simulated retinal images revealed that, although central refraction does not seem to change appreciably despite the increase in pupil diameter from 3 to $6 \mathrm{~mm}$, refractive errors estimated using the observers preferred metric
(VSMTF) tend to be more myopic than the ones predicted by the Paraxial metric for both pupil sizes, and closer to the ones predicted by the minRMS metric for a 3-mm pupil, in concordance with the results of Xu et al. ${ }^{21}$ This result seems to be inverted with the increase in pupil size. For a 6-mm pupil, minRMS predicts a large myopic shift that does not seem to correspond to the best image, according to our observer's evaluation. From the example of Fig. 4, it is also clear that the quality of the image perceived by these patients is highly dependent of pupil size and probably of the

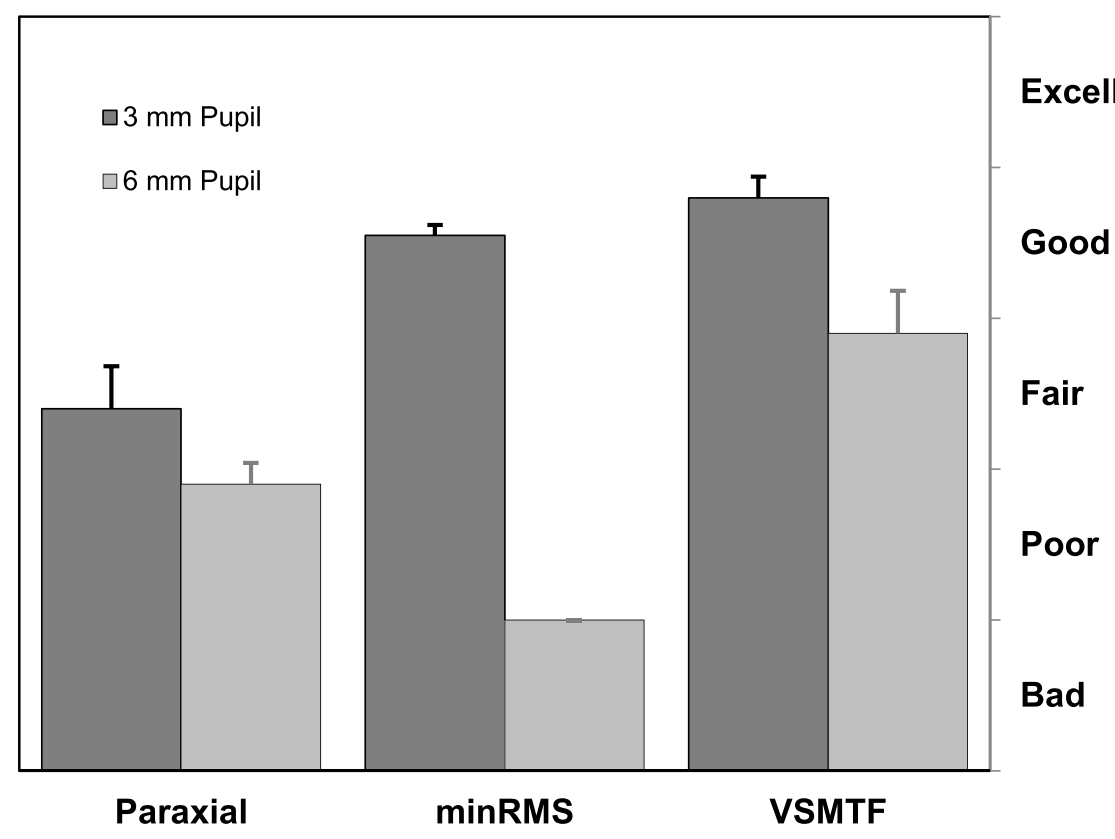

FIGURE 3.

Subjective rating of the two sets of simulated retinal images using each of the three metrics presented to three trained observers to rank image quality. minRMS, minimum root mean square error; VSMTF, visual Strehl for the modulation transfer function. 

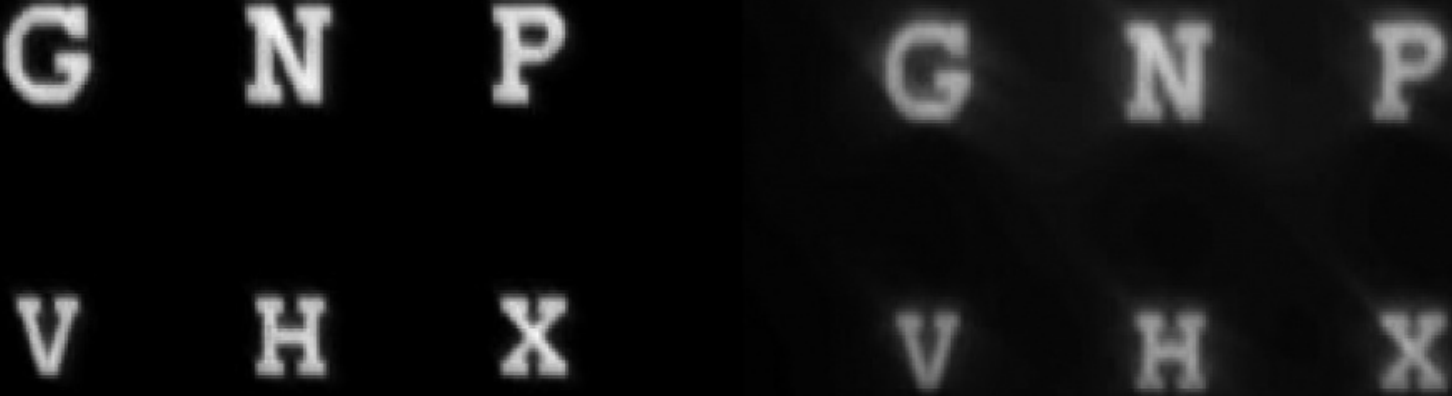

\section{FIGURE 4.}

Comparison of the foveal images simulated for a patient with 3-mm (left) and 6-mm pupil diameter (right) and their respective point spread functions (bottom).

spatial content of the visual task as well. ${ }^{45}$ Although the foveal refractive error does not seem to change appreciably with the increase in pupil diameter (VSMTF criteria), the high levels of positive spherical aberration will lead to a degradation of lower spatial frequencies, more significant under low illumination levels. It is expected that in those scenarios patients will benefit of a more negative refraction to enhance lower spatial frequencies. ${ }^{21}$ As the field angle increases, the on-axis spherical aberration becomes coma aberration, contributing to the peripheral image degradation. This and other high-order aberrations might interact to decrease image contrast in the periphery. ${ }^{46}$
The three metrics predict significantly different peripheral refraction profiles as the pupil diameter increases from 3 to $6 \mathrm{~mm}$. The subjective process used to grade the quality of the simulated foveal images corrected by each metric cannot be used for peripheral vision, but it is clear that in the presence of higher-order aberrations, the VSMTF is clearly superior to the minRMS and Paraxial metric criteria for estimating the refractive correction that maximizes visual acuity based on wavefront aberration measurements. It is then reasonable to expect that for peripheral imagery, where the pupils are nearly elliptical and higher order terms are far more significant than in fovea, visual metrics that take neural 

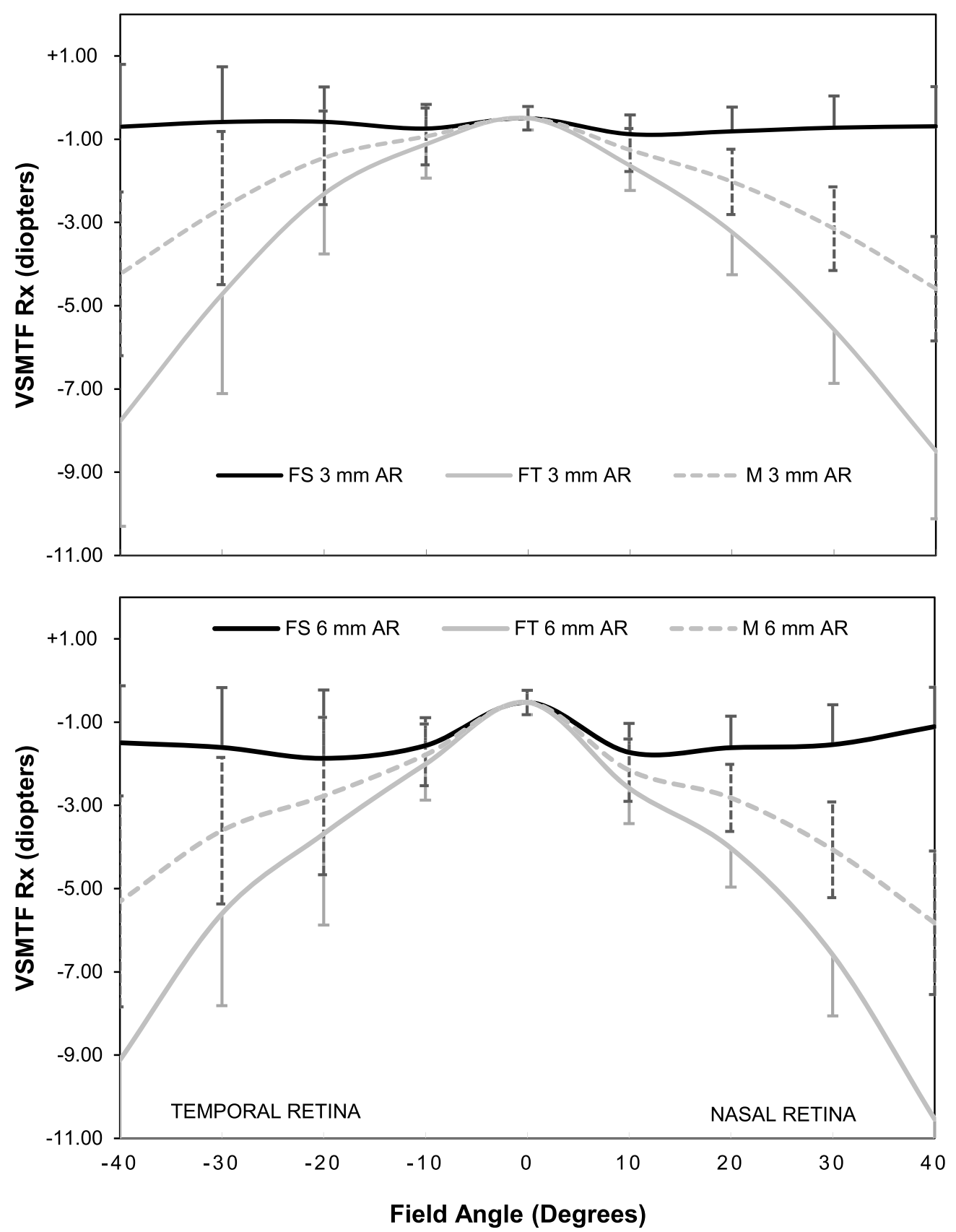

FIGURE 5.

Visual Strehl of the modulation transfer function (VSMTF) refraction calculated by substituting the symmetric retina of the Navarro eye model by the average myopic asymmetric retina (AR). Error bars represent one standard deviation. FS, sagittal power error; FT, tangential power error; M, spherical equivalent.

factors into account should also yield less biased results than metrics calculated solely based on the Zernike coefficients. That said, the hypothesis suggested by Chen ${ }^{14}$ that large pupil diameters could facilitate the effect of ortho-k to slow axial growth because of enhancement of the myopic shift in the peripheral retina seems to agree with the myopic shift observed in the VSMTF peripheral refraction profile when the pupil diameter increases from 3 to $6 \mathrm{~mm}$.

Our previous studies showed that the peripheral eye length for the average myope is shorter in the temporal retina. ${ }^{13}$ The ortho-k treatments of our sample have shown a displacement towards the temporal side of the cornea, which is in agreement with previous reports. ${ }^{47,48}$ Thus, for a light beam passing at the same distance from the center of the pupil, incident light from the nasal visual field will follow a shorter optical path towards the temporal retina, compared with the incident light from the temporal visual field. Overall, the more curved cornea and shorter eye length, and the flatter cornea and longer eye length will tend to compensate each other to render a more symmetric peripheral refraction as seen in Fig. 5.

In this case, the VSMTF metric for a 3-mm pupil diameter predicts a relative peripheral refraction profile similar to the profiles derived from measurements with the Grand Seiko open field autorefractor after ortho-k in treatments of the same degree. ${ }^{11,49-51}$ As the pupil diameter increases from 3 to $6 \mathrm{~mm}$, the average peripheral refraction profile calculated from the 
VSMTF suffers a myopic shift that can be mainly explained by the greater contribution of the paracentral zone of the treated corneas ${ }^{11}$ and the lower sensitivity to high frequencies encountered in the peripheral retina. In turn, peripheral refraction measured by the autorefractor will not take any of this changes into account. The working principle of the Grand Seiko autorefractor uses a near infrared $2.3-\mathrm{mm}$ ring-like target to illuminate the test eye and calculates second order refraction based on the size and shape of the rings' reflected image, ${ }^{52}$ making this equipment basically insensitive to the increased higher-order aberrations contribution from larger pupil diameters and to irregularities in the wavefront that lie inside the rings' area. ${ }^{16}$

In the present work, refraction obtained from the VSMTF criterion is adapted by $\mathrm{M}$-scaling to the continuously decreasing range of frequencies that are relevant with increase visual field angle compared to foveal vision. Although the metrics that would best predict peripheral refraction are not well established yet, we consider that the present approach is the more reliable and robust one as it changes the spectrum of frequencies that should be more relevant to refract the eye, as we depart from the foveal region. A limitation of this study is that our eye models are only partially customized as we do not consider the actual internal optics of each eye. A more complete personalization of the eye models $^{53}$ would require more biometric and aberrometric measurements. However, for the purpose of our study, the present approach provides a good comparison framework to evaluate the changes in axial and peripheral refraction induced by corneal reshaping with overnight ortho-k contact lenses. As the ortho-k treatment acts on the anterior corneal surface of the cornea, our results show a clearly myopic peripheral refraction that can be interpreted as the change that the treatment will induce on the whole optics of the eye.

\section{ACKNOWLEDGMENTS}

This study has been funded by FEDER through the COMPETE Program and by the Portuguese Foundation for Science and Technology (FCT) in the framework of projects PTDC/SAU-BEB/098391/2008, PTDC/SAU-BEB/ 098392/2008, and the Strategic Project PEST-C/FIS/UI607/2011. Dr. Navarro acknowledges funding by the Spanish Ministery of Economy and Competivity, grant FIS2014-58303-P. The authors have no proprietary interests in the methods and devices described in this manuscript.

Received September 29, 2015; accepted June 8, 2016.

\section{REFERENCES}

1. Lu F, Simpson T, Sorbara L, Fonn D. The relationship between the treatment zone diameter and visual, optical and subjective performance in Corneal Refractive Therapy lens wearers. Ophthalmic Physiol Opt 2007;27:568-78.

2. Queirós A, Villa-Collar C, Gutiérrez AR, Jorge J, González-Méijome JM. Quality of life of myopic subjects with different methods of visual correction using the NEI RQL-42 questionnaire. Eye Contact Lens 2012;38:116-21.

3. González-Méijome JM, Peixoto-de-Matos SC, Faria-Ribeiro M, Lopes-Ferreira DP, Jorge J, Legerton J, Queiros A. Strategies to regulate myopia progression with contact lenses: a review. Eye Contact Lens 2016;42:24-34.

4. Zhou J, Xie P, Wang D, Guo X, Yang L. The long-term clinical effects of orthokeratology in high myopia children. Zhonghua Yan Ke Za Zhi 2015;51:515-9.
5. Cho P, Cheung SW, Edwards M. The longitudinal orthokeratology research in children (LORIC) in Hong Kong: a pilot study on refractive changes and myopic control. Curr Eye Res 2005;30:71-80.

6. Charman WN. Myopia, posture and the visual environment. Ophthalmic Physiol Opt 2011;31:494-501.

7. Flitcroft DI. The complex interactions of retinal, optical and environmental factors in myopia aetiology. Prog Retin Eye Res 2012;31:622-60.

8. Kakita T, Hiraoka T, Oshika T. Influence of overnight orthokeratology on axial elongation in childhood myopia. Invest Ophthalmol Vis Sci 2011;52:2170-4.

9. Hiraoka T, Kakita T, Okamoto F, Takahashi H, Oshika T. Longterm effect of overnight orthokeratology on axial length elongation in childhood myopia: a 5-year follow-up study. Invest Ophthalmol Vis Sci 2012;53:3913-9.

10. Cho P, Cheung SW. Retardation of myopia in Orthokeratology (ROMIO) study: a 2-year randomized clinical trial. Invest Ophthalmol Vis Sci 2012;53:7077-85.

11. Queirós A, González-Méijome JM, Jorge J, Villa-Collar C, Gutiérrez AR. Peripheral refraction in myopic patients after orthokeratology. Optom Vis Sci 2010;87:323-9.

12. Faria-Ribeiro M, Queirós A, Lopes-Ferreira D, Jorge J, GonzálezMéijome JM. Peripheral refraction and retinal contour in stable and progressive myopia. Optom Vis Sci 2013;90:9-15.

13. Faria-Ribeiro M, López-Gil N, Navarro R, Lopes-Ferreira D, Jorge J, González-Méijome JM. Computing retinal contour from optical biometry. Optom Vis Sci 2014;91:430-6.

14. Chen Z, Niu L, Xue F, Qu X, Zhou Z, Zhou X, Chu R. Impact of pupil diameter on axial growth in orthokeratology. Optom Vis Sci 2012;89:1636-40.

15. Bennett JR, Stalboerger GM, Hodge DO, Schornack MM. Comparison of refractive assessment by wavefront aberrometry, autorefraction, and subjective refraction. J Optom 2015;8:109-15.

16. Bakaraju RC, Fedtke C, Ehrmann K, Ho A. Comparing the relative peripheral refraction effect of single vision and multifocal contact lenses measured using an autorefractor and an aberrometer: a pilot study. J Optom 2015;8:206-18.

17. Lebow KA, Campbell CE. A comparison of a traditional and wavefront autorefraction. Optom Vis Sci 2014;91:1191-8.

18. Thibos LN, Hong X, Bradley A, Applegate RA. Accuracy and precision of objective refraction from wavefront aberrations. $\mathrm{J}$ Vis 2004;4(4):329-51.

19. Queirós A, Villa-Collar C, González-Méijome JM, Jorge J, Gutiérrez AR. Effect of pupil size on corneal aberrations before and after standard laser in situ keratomileusis, custom laser in situ keratomileusis, and corneal refractive therapy. Am J Ophthalmol 2010;150:97-109.

20. Mathur A, Atchison DA. Effect of orthokeratology on peripheral aberrations of the eye. Optom Vis Sci 2009;86:476-84.

21. Xu R, Bradley A, Thibos LN. Impact of primary spherical aberration, spatial frequency and Stiles Crawford apodization on wavefront determined refractive error: a computational study. Ophthalmic Physiol Opt 2013;33:444-55.

22. Martin J, Vasudevan B, Himebaugh N, Bradley A, Thibos L. Unbiased estimation of refractive state of aberrated eyes. Vision Res 2011;51:1932-40.

23. Shen J, Thibos LN. Measuring ocular aberrations and image quality in peripheral vision with a clinical wavefront aberrometer. Clin Exp Optom 2009;92:212-22. 
24. González-Méijome JM, Villa-Collar C. Nomogram, corneal topography, and final prescription relations for corneal refractive therapy. Optom Vis Sci 2007;84:59-64.

25. Faria-Ribeiro M, Belsue RN, López-Gil N, González-Méijome JM. Morphology, topography, and optics of the orthokeratology cornea. J Biomed Opt 2016;21:75011.

26. Navarro R, Santamaría J, Bescós J. Accommodation-dependent model of the human eye with aspherics. J Opt Soc Am (A) 1985;2:1273-81.

27. Zemax. Optical Design Program User's Guide. Kirkland, WA: Zemax Development Corporation; 2005.

28. Navarro R, González L, Hernández JL. Optics of the average normal cornea from general and canonical representations of its surface topography. J Opt Soc Am (A) 2006;23:219-32.

29. Thibos LN, Applegate RA, Schwiegerling JT, Webb R, VSIA Standards Taskforce Members. Vision science and its applications. Standards for reporting the optical aberrations of eyes. J Refract Surg 2002;18:652-60.

30. Thibos LN. Unresolved issues in the prediction of subjective refraction from wavefront aberration maps. J Refract Surg 2004;20:S533-6.

31. Cheng X, Bradley A, Thibos LN. Predicting subjective judgment of best focus with objective image quality metrics. J Vis 2004;4(4):310-21.

32. Howarth PA, Zhang XX, Bradley A, Still DL, Thibos LN. Does the chromatic aberration of the eye vary with age? J Opt Soc Am (A) 1988;5:2087-92.

33. Campbell FW, Robson JG. Application of Fourier analysis to the visibility of gratings. J Physiol 1968;197:551-66.

34. Sachs MB, Nachmias J, Robson JG. Spatial-frequency channels in human vision. J Opt Soc Am 1971;61:1176-86.

35. Charman WN, Mathur A, Scott DH, Hartwig A, Atchison DA. Specifying peripheral aberrations in visual science. J Biomed Opt 2012;17:025004.

36. Navarro R, López JL, Díaz JA, Sinusía EP. Generalization of Zernike polynomials for regular portions of circles and ellipses. Opt Express 2014;22:21263-79.

37. Díaz JA, Navarro R. Orthonormal polynomials for elliptical wavefronts with an arbitrary orientation. Appl Opt 2014;53:2051-7.

38. Atchison DA, Scott DH, Charman WN. Measuring ocular aberrations in the peripheral visual field using Hartmann-Shack aberrometry. J Opt Soc Am (A) 2007;24:2963-73.

39. Guirao A, Williams DR. A method to predict refractive errors from wave aberration data. Optom Vis Sci 2003;80:36-42.

40. Rosén R, Lundström L, Venkataraman AP, Winter S, Unsbo P. Quick contrast sensitivity measurements in the periphery. J Vis 2014; 14:3.
41. Rovamo J, Virsu V. An estimation and application of the human cortical magnification factor. Exp Brain Res 1979;37:495-510.

42. Thibos LN, Wheeler W, Horner D. Power vectors: an application of Fourier analysis to the description and statistical analysis of refractive error. Optom Vis Sci 1997;74:367-75.

43. Rio D, Legras R. Which ratio of areas improves vision quality in simultaneous focus optics? Optom Vis Sci 2015;92:429-36.

44. Charman WN, Jennings JA, Whitefoot $H$. The refraction of the eye in the relation to spherical aberration and pupil size. Br J Physiol Opt 1978;32:78-93.

45. Liang J, Williams DR. Aberrations and retinal image quality of the normal human eye. J Opt Soc Am (A) 1997;14:2873-83.

46. de Gracia P, Dorronsoro C, Marin G, Hernández M, Marcos S. Visual acuity under combined astigmatism and coma: optical and neural adaptation effects. J Vis 2011;11:5.

47. Yang X, Gong XM, Dai ZY, Wei L, Li SX. [Topographical evaluation on decentration of orthokeratology lenses]. Zhonghua Yan Ke Za Zhi 2003;39:335-8.

48. Hiraoka T, Matsumoto Y, Okamoto F, Yamaguchi T, Hirohara Y, Mihashi T, Oshika T. Corneal higher-order aberrations induced by overnight orthokeratology. Am J Ophthalmol 2005;139:429-36.

49. Charman WN, Mountford J, Atchison DA, Markwell EL. Peripheral refraction in orthokeratology patients. Optom Vis Sci 2006;83:641-8.

50. Kang P, Swarbrick H. Peripheral refraction in myopic children wearing orthokeratology and gas-permeable lenses. Optom Vis Sci 2011;88:476-82.

51. González-Méijome JM, Faria-Ribeiro MA, Lopes-Ferreira DP, Fernandes P, Carracedo G, Queiros A. Changes in peripheral refractive profile after orthokeratology for different degrees of myopia. Curr Eye Res 2016;41:199-207.

52. Davies LN, Mallen EA, Wolffsohn JS, Gilmartin B. Clinical evaluation of the Shin-Nippon NVision-K 5001/Grand Seiko WR-5100K autorefractor. Optom Vis Sci 2003;80:320-4.

53. Navarro R, González L, Hernández-Matamoros JL. On the prediction of optical aberrations by personalized eye models. Optom Vis Sci 2006;83:371-81.

Miguel Faria-Ribeiro

Clinical \& Experimental

Optometry Research Lab (CEORLab) Centre of Physics (Optometry)

University of Minho

4710-057 Braga, Portugal

e-mail:mig.afr@gmail.com 